\title{
Glaziäre Rinnen im mittleren und nordöstlichen Niedersachsen
}

\author{
Hans Kuster \& Klaus-Dieter Meyer *)
}

Stratigraphic map (Base of Quaternary, 1:500000), Pleistocene, paleorelief, channel, fluvioglacial, lacustrine sediment, clay, marine sediment, gravel, petrographic analysis, fluvial erosion; NW-German Lowlands, Lower Saxony

Kurzfassung: Vorgelegt wird eine Quartärbasiskarte im Maßstab 1:500 000, die etwa $25000 \mathrm{~km}^{2}$ des mittleren und nördlichen Niedersachsens umfaßt.

Die Quartärbasis erscheint als stark gegliedertes Relief, bedingt durch bis über $400 \mathrm{~m}$ unter NN in das Präquartär eingeschnittene Rinnen, in denen das Quartär maximal etwa $500 \mathrm{~m}$ mächtig werden kann. Einige Rinnen lassen sich über $100 \mathrm{~km}$ weit verfolgen. Verlauf und Form der Rinnen werden durch den Untergrund modifiziert.

Die Rinnenfüllung besteht überwiegend aus Schmelzwassersedimenten sowie vor allem im Unterelbe-Unterweserbereich aus glazilimnischen Schluffen und Tonen, wovon der hangende Teil dem spätelsterzeitlichen Lauenburger Ton zuzuordnen ist. Die Überlagerung durch marine und limnische holsteinzeitliche Sedimente (die holsteinzeitliche Kieselgur der Lüneburger Heide ist in auffälliger Weise an die Rinnen gebunden) gibt eine eindeutige stratigraphische Hangendgrenze.

Grundmoräne kommt in den Rinnen relativ selten vor. Das elsterzeitliche Alter der Rinnen kann durch Geschiebezählungen nachgewiesen werden. Die aus nordischem Material bestehende Rinnenfüllung schließt eine Entstehung durch südliche Flußläufe aus. Dafür spricht auch, daß die Rinnen den Fuß der Mittelgebirge nicht erreichen. Form und Verlauf der Rinnen (Übertiefungen, steiles Gefälle und Flankenneigungen bis max. $70^{\circ}$ ) sprechen für subglazial ausgetiefte Strukturen, wobei wegen des starken Zurücktretens von Grundmoräne in tieferen Rinnenabschnitten dem Gletscherschurf nur eine untergeordnete, der subglazialen Schmelzwassertätigkeit dagegen eine dominierende Rolle zubemessen wird.

\section{[Glacial Channels in Middle and Northeastern Lower Saxony]}

A bstract: A Quaternary base map, $1: 500000$, is presented, covering about $25000 \mathrm{~km}^{2}$ of central and northeastern Lower Saxony.

The base of the Quaternary has a markedly differentiated relief due to glacial erosion channels (down to $400 \mathrm{~m}$ below present mean sea level) cut into the pre-Quaternary surface. The Quaternary sediments reach a thickness of about $500 \mathrm{~m}$ at the most. Some channels can be traced for more than $100 \mathrm{~km}$. Their course and form are affected by the conditions of the subsurface.

The channel fill consists predominantly of outwash, and mainly in the area between the lower Elbe / lower Weser of glaciolacustrine silts and clays, the overlying strata of which are to be assigned to the Late Elsterian Lauenburg Clay. The stratigraphical upper boundary is clearly marked by the overlying marine and limnic sediments of the Holsteinian (the Holsteinian kieselgur of the Lüneburg Heath is found only in the channels).

The occurrence of till is relatively rare in the channels. The Elsterian age can be ascertained by boulder counting. As the channel fill is composed of northern material, it must be excluded that the incision of channels was caused by rivers from the south. This is supported by the fact that the channels do not reach the edge of the low mountains of Northwest Germany. Shape and course of the channels (overdeepening, steep gradient and flanks up to a max. of $70^{\circ}$ ) point to subglacial deepened structures. Because of the rare occurrence of till in deeper channel sections, only little importance can be attached to the activity of glacier ploughing, whereas a major role can be ascribed to the activities of the subglacial melt water.

*) Anschrift der Verfasser: Dr. H. Kuster und Dr. K.-D. Meyer, Niedersächsisches Landesamt für Bodenforschung, Stilleweg 2, D-3000 Hannover 51. 
Inhaltsverzeichnis

1. Einleitung

2. Verbreitung und Morphologie der Rinnen

3. Füllung und Alter der Rinnen

4. Entstehung der Rinnen

5. Bemerkungen zur Karte der Quartärbasis

6. Schriftenverzeichnis

\section{Einleitung}

Seit Wolff $(1907,1909,1917)$ sind im Unterelbe- und Unterweser-Gebiet pleistozäne Rinnen bekannt. Zwar waren schon vorher, namentlich in der Hamburger Gegend, große Quartärmächtigkeiten erbohrt worden, die jedoch auf tektonische Ursachen zurückgeführt werden (GоTTSCHE 1897, 1901). WolfF dagegen erkannte deren erosive Entstehung; obgleich er an fluviatile Erosion dachte, betonte er, daß die Füllung der Rinnen rein nordischer Herkunft sei, eine Beobachtung, die manche späteren Autoren ignorierten.

Im Vergleich zu den Nachbargebieten ließ eine systematische Bearbeitung der Rinnen in Niedersachsen hauptsächlich wegen zu geringer Bohrungsdichte und nicht ausreichender Endteufe der meisten Bohrungen länger auf sich warten (vgl. u. a. W. v. Bülow 1967; Cepek 1967, 1968; Eissmann 1967, 1975; Hannemann 1964; Hannemann \& Radtke 1961 ; Heck 1939, 1963; Hinsch 1977; JohanNsen 1964; JohanNSEn \& Löhnert 1971; Johannsen \& Nachtigall 1972; Koch1924; Löhnert 1966; A. Müller 1973; WortmanN 1978). Eine von K.-D. MeYer vor knapp 20 Jahren im Rahmen hydrogeologischer Bestandsaufnahme östlich der Linie Hamburg-Soltau-Celle auf Karten im Maßstab 1:50000 erarbeitete Ubbersicht der Quartärbasis konnte deshalb nicht mehr als eine Arbeitsgrundlage sein.

Erst die Erkenntnisse aus etwa 800 - geophysikalisch vermessenen - Aufschlußbohrungen der wasserwirtschaftlichen Rahmenplanung des Larıdes Niedersachsen erlauben eine überregionale Betrachtung auch des tieferen Quartär. Mit Hilfe dieser neueren Bohrungen wurden viele ältere Bohrungen über die überwiegend flächendeckend durchgeführte Geoelektrik interpretierbar. Die geologische Betreuung der Rahmenplanbohrungen und die Auswertung der Bohrergebnisse erfolgte vor allem durch Mitarbeiter der Abteilung Hydrogeologie des NLfB. Das erste durch die wasserwirtschaftliche Rahmenplanung erkundete Gebiet war der Planungsraum „Obere-Elbe“, S und SE von Hamburg. GrobA hat hier erstmals Rinnen systematisch verfolgt und üher die Ergebnisse in mehreren Vorträgen berichtet (vgl. Groba, Ortlam \& Vierhuff 1970). Dechend, Grimme, Groba \& VIERHUFF haben 1972 eine unveröffentlichte Karte der Tiefenlage der Quartärbasis vom nördlichen Teil Niedersachsens im Maßstab 1:500 000 zusammengestellt. ORTLAM ${ }^{1}$ ) hat in einigen unveröffentlichten Berichten die Quartärbasis vor allem des nordöstlichsten Teilgebietes dargestellt. Neuere Aufschlüsse erforderten jedoch bereits eine Überarbeitung bzw. Ergänzung. Hervorzuheben sind die Beiträge von paläontologischer Seite, die vor allem Spiegler, von Daniels, Gramann und H.-J. Meyer lieferten. Auf von Daniels (1977) geht z. B. auch eine „abgedeckte“ Karte des Tertiär zurück, in die Rinnen - vorwiegend aufgrund von Bohrergebnissen der wasserwirtschaftlichen Rahmenplanung - eingetragen sind.

KuSTER, der die meisten Bohrungen der wasserwirtschaftlichen Rahmenplanung zwischen Elbe und Weser betreute und weiter bearbeitet, hat 1973 eine unveröffentlichte de-

1) Die Veröffentlichung von Ortlam \& Vierhuff (1978): Aspekte zur Geologie des höheren Känozoikums zwischen Elbe und Weser-Aller. - N. Jb. Geol. Paläont. Mh. 1978 (7): 408-426, 7 Abb., 1 Tab.; Stuttgart. - erschien erst nach Abschluß des Manuskripts vorliegender Arbeit. 
taillierte Quartärbasiskarte des Raumes Stade-Zeven-Bremervörde zusammengestellt, die laufend erweitert und auf den neuesten Stand gebracht wurde. Hieran war neben den Diplom-Geologen Hultquist, Schwerdtfeger und Wafai auch Herr Ing.-grad. GRIMME beteiligt, dem die Autoren darüber hinaus manche andere wertvolle Unterstützung verdanken.

Für die beiliegende Quartärbasiskarte standen folgende als Beikarten zur GK 25 erarbeiteten Quartärbasiskarten zur Verfügung: 3320 Liebenau, 3420 Stolzenau, $3421 \mathrm{Hu}-$ sum, 3422 Neustadt am Rübenberge, 3521 Rehburg, 3522 Wunstorf und 3523 Stöcken. Diese Blätter ergaben zusammen mit den bereits publizierten Blättern der GK 253424 Mellendorf, 3524 Isernhagen und 3524 Großburgwedel ein sehr detailliertes Bild der Quartärbasis des Raumes nördlich von Hannover. Ergänzende Daten für die nordrheinwestfälischen Gebiete im SW der Karte lieferte freundlicherweise Herr Dr. DAHM, Geologisches Landesamt Krefeld.

Allen, die zum Gelingen vorliegender Arbeit beigetragen haben, sagen wir herzlichen Dank. Wir denken dabei auch an alle nicht namentlich genannten Kollegen und Mitarbeiter sowie die beteiligten Vertreter der einzelnen Wasserwirtschaftsämter und Bohrsowie Geomeßfirmen.

\section{Verbreitung und Morphologie der Rinnen}

Für die relativ schmalen, länglichen Formen hat sich seit WolfF (1907) die Bezeichnung „Rinnen“ eingebürgert, womit im N des Gebietes Eintiefungen etwa ab - $100 \mathrm{~m}$ NN verstanden werden (voN BüLow 1967: 407), während im Bergvorland auch von wenig mehr als $10 \mathrm{~m}$ Tiefe bei einer Höhenlage teilweise über NN als Rinnen bezeichnet werden (WORTMANN 1968).

Einen Uberblick über Anzahl, Verlauf, Breite und Tiefe der Rinnen vermittelt die beiliegende Karte (Kt. 1).

Südlich einer Linie Gifhorn-Celle-Hoya-Delmenhorsî liegt die Quartärbasis meist nicht tiefer als $25 \mathrm{~m}$ unter NN. Entsprechend der Morphologie steigt die Quartärbasis großflächig nach $S$ an. Hier verlaufen Linien gleicher Tiefenlage bis max. $25 \mathrm{~m}$ unter NN indifferent oder sind ost-westlich ausgerichtet. Bei den selten erreichten Tiefen von mehr als $50 \mathrm{~m}$ unter $\mathrm{NN}$ ist aber die N-S-Richtung angedeutet, die nördlich der genannten Linie dominiert. Einige der markantesten N-S-verlaufenden Rinnen lassen sich über $100 \mathrm{~km}$ bis zur Elbe verfolgen. Sie sind z. T. über $400 \mathrm{~m}$ tief in den tertiären Untergrund eingeschnitten.

Die größte bis heute erbohrte Quartär-Mächtigkeit südlich der Elbe ist aus der „ReeBelner Rinne“ mit 502 m (= 434 m unter NN; vgl. LütTig 1972: Abb. 4) bekannt, die im Bereich der Karte NW-SE verläuft.

Den markantesten, meist deutlich linearen Vertiefungen sind Flächen extremer Hochlage oft unmittelbar benachbart (z. B. bei Uelzen, in der Nordheide).

Die Rinnen erinnern nach ihrer Form an verschiedene glaziäre Ausräumungsstrukiuren im mitteleuropäischen Vereisungsgebiet, wie sie Eissmann (1967) darstellte. Es kommen bei vergleichbarer Eintiefung extrem schmale $(<500 \mathrm{~m}$; Nord- und Südheide) bis beckenförmige Gebilde vor ( $>4000 \mathrm{~m}$; Bremervörde, Hoya, Syke).

Anhand eines Querprofils durch die dicht abgebohrte "Wintermoorer Rinne“ in der Nordheide (BüCHNER 1971) läßt sich ein Neigungswinkel einer Rinnenflanke von $55^{\circ}$ ermitteln. In derselben Rinne ergab sich nördlich Buxtehude bei nur $10 \mathrm{~m}$ entfernten Bohrungen eine Differenz der Tiefenlage der Quartärbasis von rund $30 \mathrm{~m}$. Dies bedeutet ein 


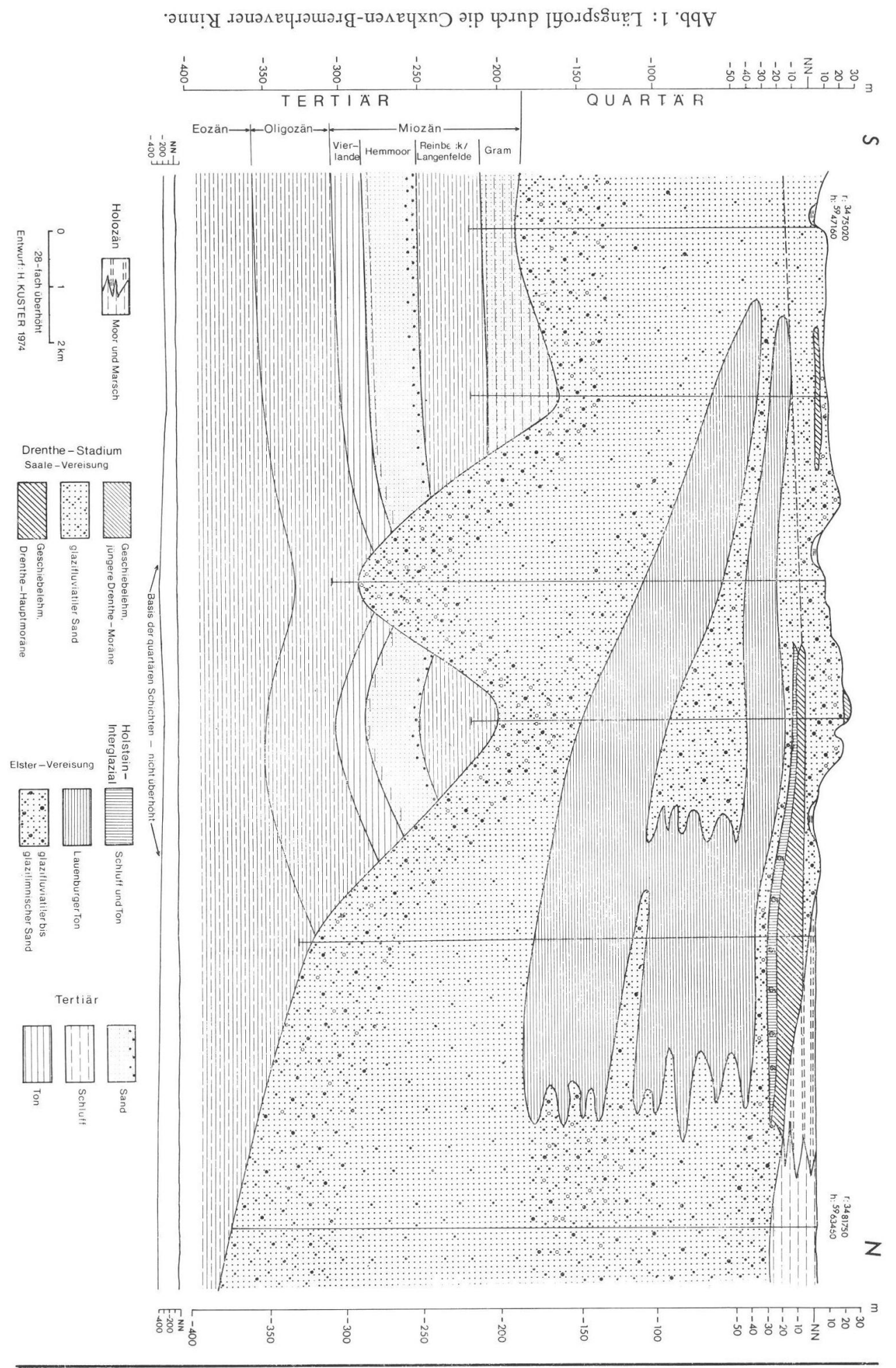




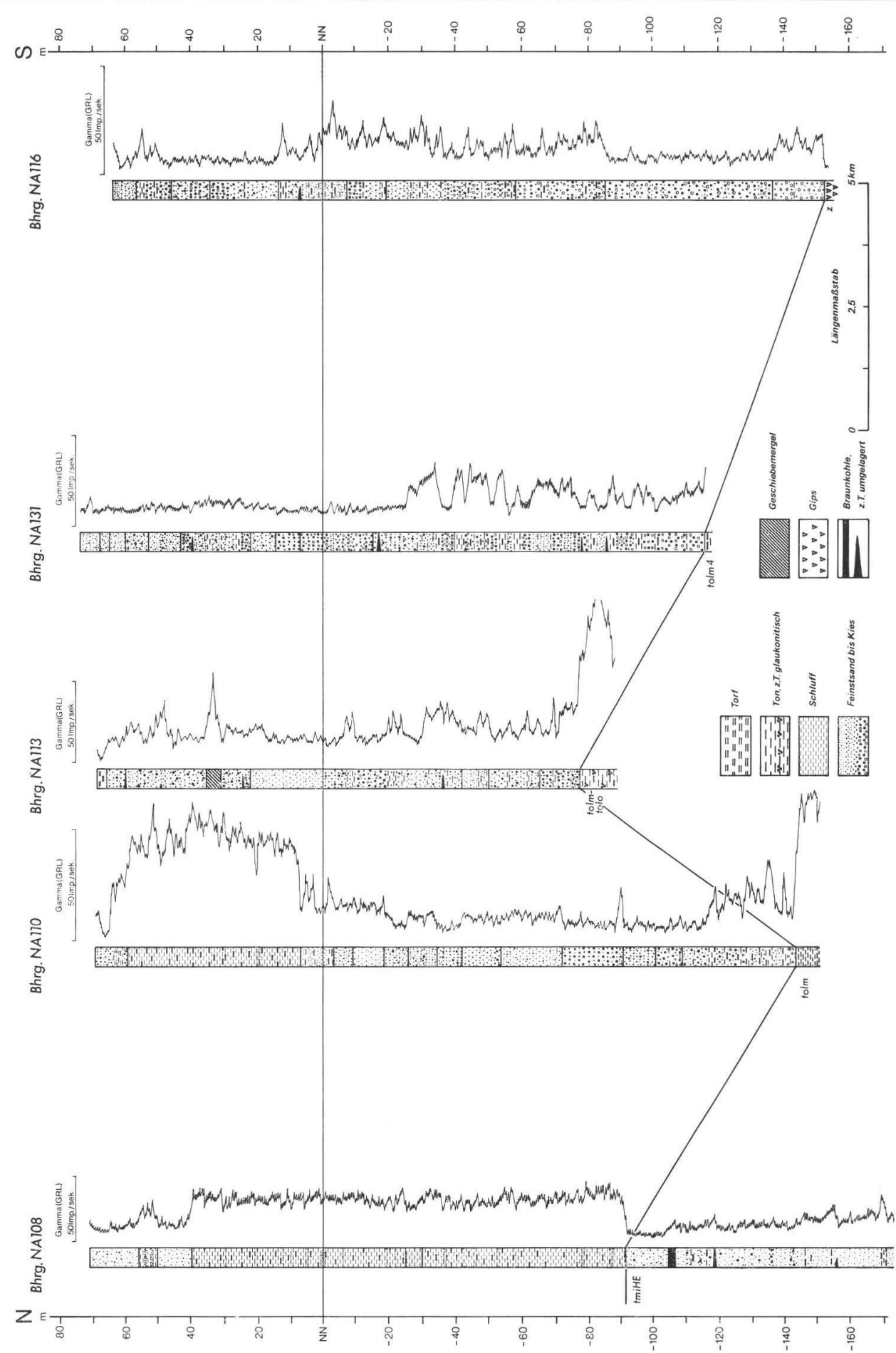

Abb. 2: Bohrungen in der Wietzendorf-Bonstorfer Rinne (Gamma-Ray-Logs nach den Bohrlochmeßdiagrammen der Tegtmeyer-Geophysik GmbH. Präquartär: $z=$ Zechstein, tol = Oligozän, tmiHE $=$ Miozän, Hemmoor-Stufe. 
Gefälle von etwa $70^{\circ}$. Normalerweise dürften die Rinnenflanken, wie man z. B. an geoelektrischen Querprofilen erkennen kann, jedoch flacher sein. Der Rinnenboden ist gewellt. Zumindest in der Längsrichtung zeichnen sich deutliche Höhenunterschiede ab (s. Abb. 1 und 2).

Interessant sind die Beziehungen zum heutigen Flußnetz, welches stellenweise durch die Rinnen beeinflußt erscheint. Die bevorzugte Richtung der Hauptvorfluter Elbe und Weser-Aller, NW-SE, ist bei den Rinnen nordwestlich Celle und im NE des Arbeitsgebietes, nahe der Elbe, erkennbar. Sichtlich ohne Beeinflussung quert andererseits die Weser einige größere Rinnen.

Einen weitgehend von den tieferen Strukturen im Gebiet des Steinhuder Meeres unabhängigen Verlauf zeigt auch die Leine (Jordan \& Voss 1978). Dagegen scheinen die untergeordneten Vorfluter Teilstrecken von Rinnen zu folgen (z. B. Oste, Wümme, Schwinge, Este, Seeve-Hanstedter Aue).

Verbreitung, Verlauf und evtl. die Form der Rinnen sind vermutlich durch den Untergrund modifiziert. Es ist auffällig, daß gerade dort Rinnen nur spärlich auftreten bzw. meist nur relativ flach sind, wo heute unmittelbar unter dem Quartär mächtiger obermiozäner Glimmerton verbreitet ist (mittlerer Teil des Arbeitsgebietes; im NE = Braunkohlensande, im NW = obermiozäne-pliozäne Fein-/Feinstsande).

Daneben drängt sich die Vorstellung auf, daß im S das auftauchende tonig-schluffige Untermiozän und Oligozän als Barriere fungiert hat.

Salzstöcke und Salzmauern üben ihren Einfluß direkt sowie indirekt über ihre Randsenken und/oder ihre angehobene Bedeckung aus. Tatsächlich verlaufen tiefe Rinnen durch sämtliche Randsenken, die noch im Jungtertiär besonders mobil waren (Stader, Zevener, Soltauer, Dethlinger, Lüneburger, Dannenberger Becken). Salzstöcke in Niveaus zwischen 50 bis $250 \mathrm{~m}$ unter NN scheinen besondere „Anziehungspunkte“ für Rinnen gewesen zu sein. So wurden z. B. die Strukturen Bramel, Hamwiede, Soltau, Dethlingen, Sülze, Lutterloh und Bokel von Rinnen durchquert, die jeweils bis in den Gipshut eingetieft sind.

Eine besondere Rolle spielen offenbar die drei letztgenannten Strukturen. Auf diesen enden Rinnen ziemlich abrupt, nachdem sie kurz vorher noch tief eingeschnitten sind. Auf dem Salzstock Sülze erreicht die betreffende Rinne (Abb. 2 und 3) ihre maximale Teufe überhaupt. Es ist wohl kein Zufall, daß diese Salzstöcke alle am Nordrand der oben genannten Barriere liegen.

Andere Salzstöcke extremer Hochlage sind wohl durch die schützende Hülle aus aufgestauchtem Deckgebirge von Rinnen verschont geblieben (Stade, Lüneburg). Vorstellbar wäre, daß die N-S verlaufenden Salzmauern im NW zumindest da, wo sie hartes Material (z. B. Kreidekalkstein bei Hemmoor) in Oberflächennähe gehoben haben, die Richtung der Rinnen beeinflußt haben oder daß sie eventuell für die breite Form der Rinnen in diesem Bereich mitverantwortlich sind.

\section{Füllung und Alter der Rinnen}

Die Rinnen sind mit Sedimenten der unterschiedlichsten Korngrößen gefüllt. Neben über $200 \mathrm{~m}$ mächtigen Grob-/Mittel-Sanden und Kiesen, z. T. ohne jede Zwischenlage von feiner-körnigem Material, finden sich z. B. in relativ kurzem horizontalen Abstand ausschließlich Feinstsande, Schluffe oder Wechsellagerungen aller genannten Sedimente. Einigermaßen typisch sind sehr schlecht sortierte Korngemische. Fast charakteristisch ist speziell für tiefere Rinnen eine Geschiebelage an der Basis. Als Beispiel sei auf die CuxhavenBremerhavener Rinne verwiesen mit einer bis zu mehr als $50 \mathrm{~m}$ mächtigen Geröllpackung, die örtlich fast ausschließlich aus hellgrünlich-grauem Eozän-Gestein besteht (Abb. 1). 
Die gröberen Basispartien spielen für die Wasserversorgung in verschiedenen Gebieten eine große Rolle. Diesem Umstand verdanken wir z. B. auch die Kiesproben aus Bohrungen für den Brunnen III des Wasserwerkes Adendorf bei Lüneburg (Tab. 1).

Die Art der Füllung scheint nicht unabhängig von der äußeren Form einer Rinne zu sein. Rinnen zeigen dort den grobkörnigsten Inhalt, wo sie am engsten und gleichzeitig oft auch am tiefsten eingeschnitten sind. Andererseits scheint sich auch ähnlich wie bei fluviatilen Verhältnissen die Krümmung auf die Sedimentation auszuwirken. So gibt es manchen Hinweis für gröbere Ablagerungen am „Prall“- und umgekehrt feinere am „Gleit"-Hang.

\begin{tabular}{|c|c|c|c|c|c|}
\hline Probe-Nummer: & 1 & 2 & 3 & 4 & 5 \\
\hline Teufe $[\mathrm{m}]:$ & $265-285$ & $304-307$ & $276-278$ & $290-293$ & $301-302$ \\
\hline Nordische Komponente: & & & & & \\
\hline Kristallin (K) & 360 & 1859 & 359 & 1340 & 809 \\
\hline Sediment (S) & 184 & 1150 & 182 & 881 & 385 \\
\hline Kalk, grau $\}^{(P K)}$ & 74 & 105 & 38 & 124 & 74 \\
\hline Kalk, schwarz & 1 & 6 & & & 3 \\
\hline Kalk, rot J & 6 & 17 & 12 & 20 & 7 \\
\hline Kreidekalk (Kk) & & 6 & & & \\
\hline Flint $(F)$ & 518 & 2288 & 318 & 1354 & 557 \\
\hline Quarz (Q) & 5 & 26 & 10 & 51 & 12 \\
\hline $\begin{array}{l}\text { Lokale Komponente: } \\
\text { (meist tertiär) }\end{array}$ & & & & & \\
\hline Sandstein & 25 & 288 & 40 & 145 & 225 \\
\hline Tonstein & & 20 & 15 & 12 & 17 \\
\hline Braunkohle & 37 & 26 & 6 & & 4 \\
\hline Kieselholz & & 1 & & & 3 \\
\hline Pyrit & 6 & 37 & 5 & 38 & 14 \\
\hline Faserka1k & 1 & 3 & 2 & 4 & 1 \\
\hline Kieselgestein & & & & 1 & \\
\hline Schill & 56 & 43 & & 27 & 1 \\
\hline Südliche Komponente: & & & & & \\
\hline Lydit & & 1 & & & \\
\hline Summe: & 1273 & 3876 & 987 & 3997 & 2113 \\
\hline TGZ & $\begin{array}{ll}\lambda & 14,86 \\
\rho & 58,23\end{array}$ & $\begin{array}{l}14,30 \\
58,88\end{array}$ & $\begin{array}{l}14,80 \\
58,07\end{array}$ & $\begin{array}{l}14,82 \\
58,71\end{array}$ & $\begin{array}{l}14,73 \\
58,46\end{array}$ \\
\hline$Q: K$ & 0,01 & 0,01 & 0,03 & 0,04 & 0,02 \\
\hline $\mathrm{F}: \mathrm{K}$ & 1,43 & 1,23 & 0,89 & 1,01 & 0,69 \\
\hline $\mathrm{S}: \mathrm{K}$ & 0,51 & 0,62 & 0,51 & 0,66 & 0,48 \\
\hline $\mathrm{PK}: \mathrm{K}$ & 0,23 & 0,07 & 0,14 & 0,11 & 0,10 \\
\hline $\mathrm{PK}: \mathrm{S}$ & 0,44 & 0,11 & 0,27 & 0,16 & 0,22 \\
\hline$F: P K$ & 6,40 & 17,88 & 6,36 & 9,40 & 6,63 \\
\hline
\end{tabular}

Tab. 1: Geschiebezählungen elsterzeitlicher Kiese Brunnen III Adendorf. Fraktion 15-60 mm, TK 25 Lüneburg (2728) R: 3598 14, H: 5906 75; Geländeoberfläche 28,5 m, Pr. 1-2 alte Bohrung, Pr. 3-5 neue Bohrung; Bohrzeit 1968, Analyse: K.-D. Meyer. 
Die Rinnen differieren nach ihrer Füllung nicht nur in sich selbst, sondern unterscheiden sich auch untereinander. So ist z. B. die Anhäufung des Eozän-Gesteins in der Cuxhaven-Bremerhavener Rinne aus keiner anderen Erosionsform, auch nicht aus der benachbarten ähnlich tiefen Oxstedter Rinne, bis jetzt bekannt geworden.

Sehr unterschiedlich gefüllt sind zwei über $40 \mathrm{~km}$ parallel verlaufende, nur etwa $5 \mathrm{~km}$ voneinander entfernte Rinnen. Während Teilstrecken der östlichen, der Rotenburger Rinne, die als Grundwasserlieferant besonders geschätzt wird, schon länger bekannt sind, blieb die westlich benachbarte, die lokal noch tiefer eingeschnitten ist, wegen ihrer vorwiegend schluffig/feinsandigen Füllung bis vor kurzem unentdeckt. Erst im gemeinsamen Bett südlich Verden herrschen gröberkörnige Sedimente vor, ähnlich denen in der nördlichen Rotenburger Rinne.

Ahnlich wie auf Abb. 1, 2 und 4 dargestellt, finden sich in tieferen Rinnen meistens im mittleren bis höheren Teil mächtigere Pakete aus vorwiegend feinsandigem, olivgrauen bis grauschwarzen Schluff bis Ton, dem Lauenburger Ton. Bei homogener sandarmer Ausbildung kann er - makroskopisch - eine frappierende A hnlichkeit mit dem obermiozänen Glimmerton haben. Meist ist jedoch der Kalkgehalt des Lauenburger Tons beträchtlich höher. Für letzteren ist eine „Mischfauna“ typisch. So hat z. B. SpIEgler in Proben aus dem Lauenburger Ton in der Bohrung NA 108 Meinholz (Abb. 2) neben KreideBryozoen, Foraminiferen aus der Kreide sowie vorwiegend aus dem Neochatt nachgewiesen. In Proben eines etwa 90 m mächtigen "quartären" tonigen Schluffs aus der Bohrung UWO 54 Ostervesede wurden - ebenfalls nach SPIEGLER - Foraminiferen der Kreide und vieler im Nordwestdeutschen Becken verbreiteten Tertiärstufen gefunden.

Die größte Mächtigkeit von „kompaktem“ Lauenburger Ton wurde bisher mit mehr als $160 \mathrm{~m}$ in der Bohrung UWO 149 Neuenkirchen nachgewiesen. In der Reeßelner Rinne (Bohrung HWW 91 Reeßeln) erreicht er $150 \mathrm{~m}$. Mit sandigen Zwischenlagen wird der Lauenburger Ton in der UWO 6 Wedehof $170 \mathrm{~m}$ mächtig. Auch in weniger tiefen Rinnen wird der Lauenburger Ton in vorwiegend tonig-schluffiger Fazies dicker als $100 \mathrm{~m}$. So repräsentiert er in der Wietzendorf-Bonstorfer Rinne mit rund $130 \mathrm{~m}$ lokal fast die komplette Füllung (Abb. 2). Diese relativ homogene Ausbildung des Lauenburger Tons ist jedoch nur eine seiner Erscheinungsarten. Wegen des raschen lateralen und vertikalen Fazieswechsels zwischen Ton, Schluff und Sand - wie in der Typregion (Meyer 1965) sollte man denn auch besser von dem „Komplex des Lauenburger Tons“ sprechen. Wahrscheinlich spielte Toteis in den Rinnen eine große Rolle, so daß es nicht zu einer gleichmäßigen Tonsedimentation kam, d. h. es blieben große Abschnitte während der Abschmelzphase von der wahrscheinlich rasch erfolgenden Grobsedimentation verschont.

Noch während des Holstein-Interglazials müssen Rinnen partiell als Hohlformen existiert haben. So konnte in Küstennähe das Holsteinmeer ingredieren (Abb. 1), während sich in der Lüneburger Heide im Bereich verschiedener Rinnen Kieselgur bildete (Abb. 3). Die großen bekannten Lagerstätten aus dieser Zeit (Hetendorf-Bonstorf, Breloh-Munster und Oberohe-Wiechel) sind eindeutig an die Flanken von Rinnen gebunden. Diese Erkenntnis half z. B. beim Verfolgen der sehr schmalen Rinne zwischen Bonstorf und Hermannsburg. Auch ein paar Vorkommen fraglichen Alters liegen in ähnlicher Position.

Spätestens während des Drenthe-Stadiums der Saale-Eiszeit jedoch wurde z. B. die Rinne, die bei Bispingen von der heutigen Luhe gequert wird, völlig verfüllt. Denn die eemzeitliche Kieselgur hält sich an das Luhetal, das $\mathrm{nicht}$ durch die genannte Rinne vorgezeichnet erscheint.

Mit dem spätelsterzeitlichen Lauenburger Ton und der darüber folgenden marinen Ingression des Holstein-Interglazials ist also eine zeitliche obere Fixierung gegeben. Wo diese Sedimente fehlen, wäre theoretisch auch ein jüngeres Alter möglich; bis jetzt aber 


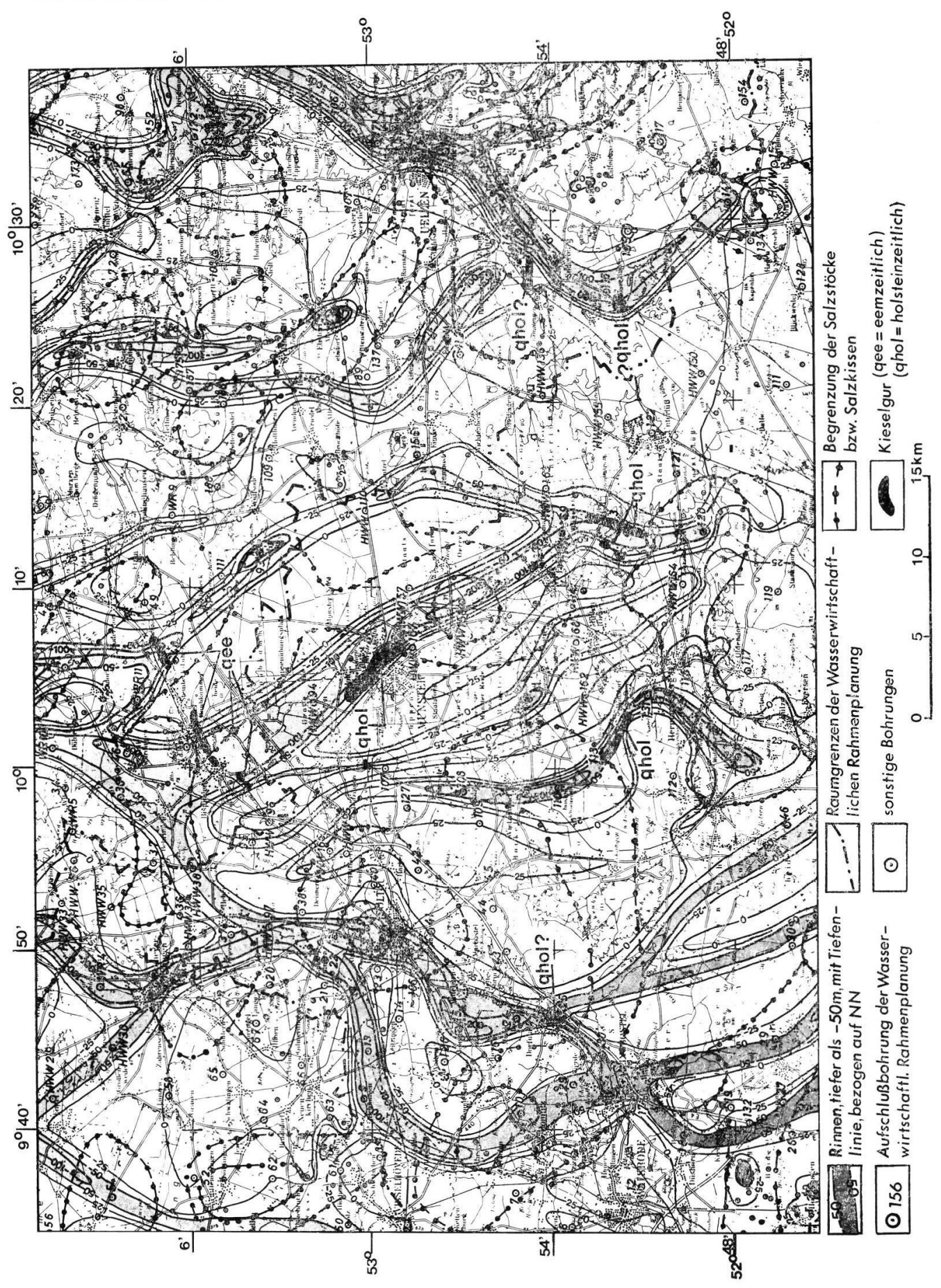

Abb. 3: Quartärbasis und Kieselgurvorkommen im Gebiet Soltau-Munster-Uelzen. (Kieselgurvorkommen nach BENDA \& BRANDES 1974). 


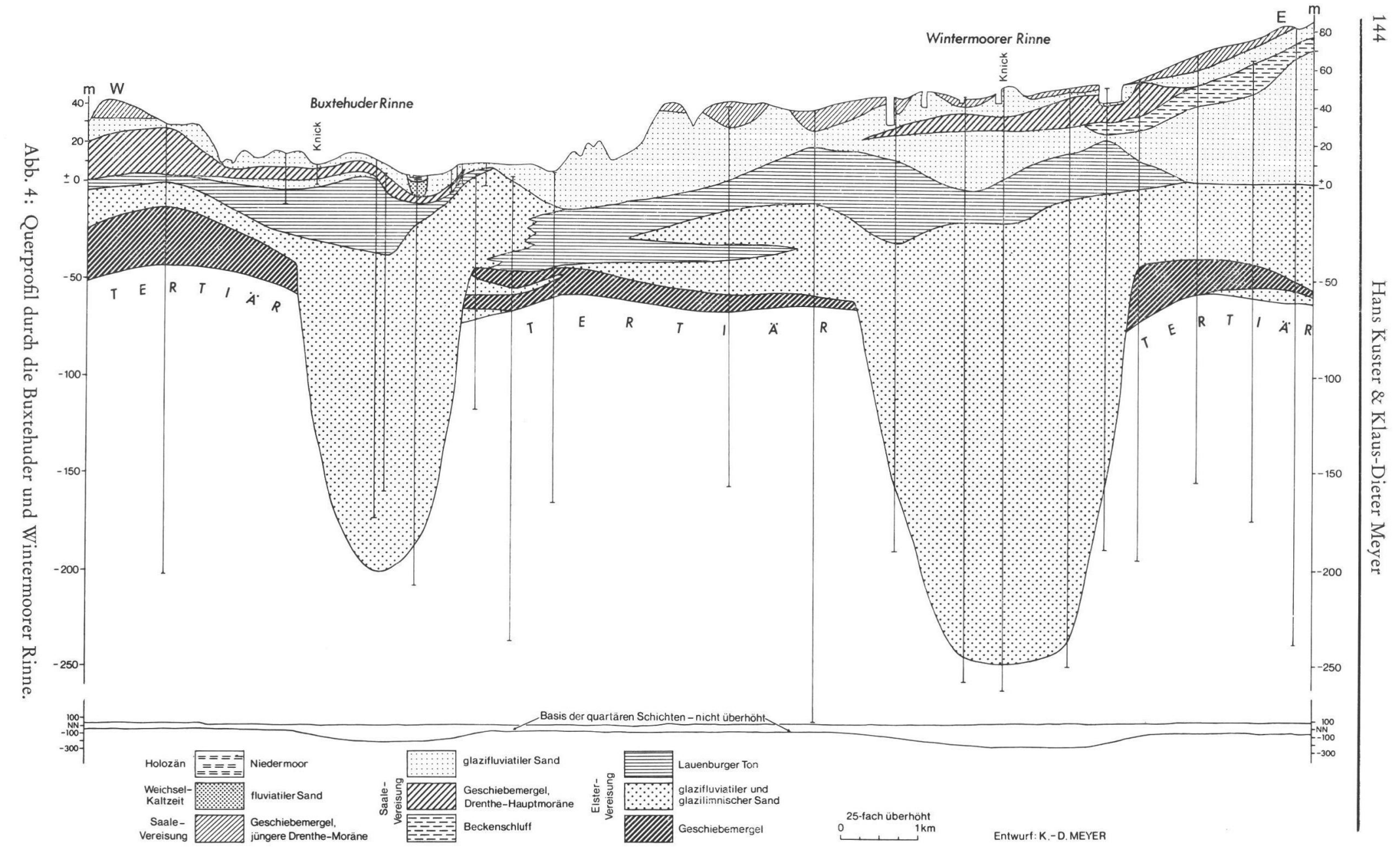


gibt es aus Niedersachsen und Hamburg (Grube \& EhLERs 1975) keine Beweise für saalezeitliche Rinnen derartigen Ausmaßes.

Auffallend ist, daß Geschiebemergel in den Rinnen eine untergeordnete Rolle spielt, wobei er in tieferen Abschnitten der Rinnen-Bohrungen meist völlig fehlt. Letzteres ist z. B. der Fall in dem auf Abb. 4 dargestellten Querschnitt. Die sehr gleichmäßig ausgebildete Elster-Moräne ( $z$. T. in 2 Bänke aufgespalten) geht hier nicht in die Rinnen hinein, sondern wird abrupt von diesen abgeschnitten. In anderen Fällen zieht sich die Moräne zwar etwas auf die „Rinnenschulter" herab, fehlt im zentralen Teil jedoch wieder (z. B. UE 45, UWO 85; UE 48, UE 49). Andererseits ist von Grube \& EHLERs (1975) ein Profil aus Hamburg veröffentlicht worden, in dem die Elster-Moräne - leicht durchhängend - die gesamte Rinne durchzieht. Wie weitere Beispiele aus dem Hamburger Raum bezeugen, scheint das aber eher die Ausnahme zu sein. Dies zeigen bereits die Profile auf den Blättern Nr. 2424 Wedel und 2425 Hamburg der GK 25 (Wolff 1913, 1914).

Wenn die Grundmoräne in die Rinnen hineinzieht, dann beschränkt sie sich häufig nur auf die obere Hälfte; nicht selten ist sie dann in mehrere, nicht korrelierbare Bänke aufgespalten. Besonders bei tieferen Geschiebemergelvorkommen ist deren Grundmoränencharakter nicht immer zweifelsfrei. Es könnte sich z. B. auch um mit Kies durchsetzte Beckensedimente, subaquatische Moränen oder Versturzbildungen handeln (GRIPP 1956), die u. a. zu Schichtverdoppelungen führten. Ferner können mit dem tonig-schluffigen Untergrund verunreinigte kiesige Basispartien eine basale Grundmoräne vortäuschen. Man kann außerdem feststellen, daß selbst dort, wo Grundmoräne verhältnismäßig tief (200 bis $300 \mathrm{~m}$ ) liegt, diese meist nicht direkt dem Präquartär auflagert, sondern von diesem noch durch mehrere $\mathrm{m}$ bis zehner-m sandig-kiesigen Materials getrennt ist. Dabei scheinen die Fälle mit Grundmoräne an oder nahe der Basis auf die breiteren Strukturen beschränkt, die eher Übergänge zu Zungenbecken vermuten lassen.

Wie im Geschiebemergel und im Lauenburger Ton spiegelt sich innerhalb einer Rinne das Substrat auch in den Sanden wider. Unschwer lassen sich z. B. im NE umgelagerte miozäne Braunkohlensande anhand der gut gerundeten, z. T. bläulichen Quarze erkennen. Makrofossilien aus dem marinen Hemmoor sind vielfach so unversehrt, daß sie eigentlich nur aus dem unmittelbar benachbarten Rinnenufer stammen können. Daneben läßt sich in den basalen Grobserien häufig schluffig/toniges Material des Untergrundes identifizieren. Im Bereich zwischen Delmenhorst und Hoya bestehen die tieferen Teile der Rinnenfüllung örtlich fast ausschließlich aus umgelagerten tertiären Sedimenten (z. B. Glaukonitsande). Weiterhin sind noch Schollen tertiärer vorwiegend tonig/schluffiger Serien zu erwähnen.

Soweit möglich, wurde die petrographische Zusammensetzung der Kiese namentlich aus den tieferen Rinnenabschnitten untersucht, wobei meist nur die Feinkiesfraktion zur Verfügung stand, seltener auch ausreichendes Material der Mittel- und Grobkiesfraktion.

Es zeigte sich (Tab. 1 und 2), daß die Kiese, abgesehen von wechselnden Anteilen lokalen Materials aus den Rinnenflanken und dem Untergrund (in erster Linie TertiärSandstein, Tonstein, Pyrit, Lignit, Schill; seltener Oberkreide) praktisch ausschließlich nordischer Herkunft sind. Diese zuerst von WolfF im Bremer und Hamburger Raum gemachte Beobachtung kann also verallgemeinert werden. Das Material (hauptsächlich die nordischen paläozoischen und kretazischen Kalksteine) ist meistens frisch und unverwittert. Ein pliozänes oder auch ältestpleistozänes („präglaziales“) Alter der Rinnen ist damit ausgeschlossen, denn eine totale spätere Ausräumung ist unwahrscheinlich. Ist somit die Rinnenfüllung eindeutig glazigenen Ursprungs und sind deren höhere Abschnitte als elsterzeitlich gesichert, so geht ein elsterzeitliches Alter auch für die tieferen Abschnitte daraus nicht einfach hervor. CEPEK (1967) nimmt an, daß die tieferen Grundmoränen in 


\begin{tabular}{|c|c|c|c|c|c|c|c|c|c|c|c|c|c|c|c|c|c|c|c|c|c|c|c|c|}
\hline Bohrung & TK 25 & & $\mathbf{R}$ & & & $\mathrm{H}$ & & GO & $q-N N$ & $\begin{array}{l}\text { Teufe } \\
\text { in } \mathrm{m}\end{array}$ & Material & $\mathrm{K}$ & $\mathrm{s}$ & $\mathrm{F}$ & PK & Q & $\mathrm{Q}: \mathrm{K}$ & $\mathrm{F}: \mathrm{K}$ & |S:K $\mid$ & PK:K & PK:S & $F: P K$ & Summe & $\begin{array}{l}\text { Loka1- } \\
\text { Geschiebe }\end{array}$ \\
\hline UE 97 & 2420 & 35 & 002 & 240 & 59 & 304 & 450 & 19 & -112 & $99-102$ & $\mathrm{qe} / / \mathrm{gf}$ & 50 & 21 & 11 & 13 & 5 & 0,11 & 0,22 & 0,42 & 0,25 & 0,60 & 0,86 & 333 & 1 \\
\hline " & $"$ & & $"$ & & & $"$ & & $"$ & $"$ & $102-105$ & & 43 & 26 & 11 & 11 & 9 & 0,20 & 0,27 & 0,62 & 0,26 & 0,42 & 1,0 & 380 & 1 \\
\hline$"$ & $"$ & & $"$ & & & $"$ & & $"$ & " & $105-108$ & $"$ & 43 & 22 & 9 & 15 & 11 & 0,27 & 0,21 & 0,52 & 0,37 & 0,69 & 0,58 & 436 & 3 \\
\hline " & " & & $"$ & & & $"$ & & " & " & $108-111$ & $"$ & 43 & 22 & 9 & 16 & 10 & 0,23 & 0,20 & 0,51 & 0,37 & 0,72 & 0,53 & 508 & 2 \\
\hline$"$ & $"$ & & " & & & $"$ & & " & $"$ & $111-114$ & $"$ & 41 & 20 & 7 & 24 & 8 & 0,20 & 0,18 & 0,50 & 0,58 & 1,17 & 0,32 & 415 & - \\
\hline$"$ & $"$ & & $"$ & & & " & & $"$ & $"$ & $114-117$ & $"$ & 36 & 28 & 8 & 19 & 10 & 0,28 & 0,22 & 0,78 & 0,53 & 0,68 & 0,41 & 477 & 4 \\
\hline$"$ & $"$ & & " & & & $"$ & & $"$ & $"$ & $117-120$ & " & 40 & 25 & 9 & 18 & 7 & 0,17 & 0,22 & 0,64 & 0,47 & 0,73 & 0,47 & 578 & 1 \\
\hline UE 111 & 2118 & 34 & 817 & 750 & 59 & 634 & 460 & 1 & -374 & $366-369$ & $"$ & 30 & 31 & 25 & 12 & 2 & 0,07 & 0,81 & 1,03 & 0,4 & 0,39 & $\begin{array}{l}9 \\
9\end{array}$ & 497 & - \\
\hline UE 133 & 2422 & 35 & $26 \mathrm{c}$ & 080 & 59 & 342 & 200 & 6 & -127 & $129-135$ & " & 45 & 14 & 14 & 21 & 4 & 0,09 & 0,31 & 0,32 & 0,45 & 1,51 & 0,65 & 403 & $\left.3^{x}\right)$ \\
\hline UE 136 & 2423 & 35 & 35 & 700 & 59 & 35 & 960 & 25 & -195 & $81-84$ & $"$ & 45 & 18 & 15 & 20 & 2 & 0,05 & 0,32 & 0,39 & 0,44 & 1,14 & 0,73 & 444 & - \\
\hline A 15 & 2422 & 35 & 23 & 950 & 59 & 30 & 490 & 25 & -165 & $189-190$ & " & 30 & 21 & 49 & - & - & - & 1,64 & 0,68 & - & - & - & 146 & - \\
\hline C III & 2920 & 35 & 05 & 445 & 58 & 767 & 715 & 20 & $<-132$ & $8-18$ & $\mathrm{qD} / / \mathrm{gf}$ & 46 & 17 & 12 & 18 & 5 & 0,11 & 0,26 & 0,38 & 0,39 & 1,02 & 0,68 & 246 & - \\
\hline 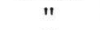 & " & & " & & & & & " & . & $101-106$ & $\mathrm{qe} / / \mathrm{gf}$ & 47 & 20 & 15 & 8 & 10 & 0,22 & 0,32 & 0,43 & 0,18 & 0,42 & $2 \mid 1,81$ & 373 & - \\
\hline " & $"$ & & " & & & " & & " & $"$ & $148-150$ & $"$ & 51 & 21 & 17 & 6 & 5 & 0,10 & 0,33 & 0,40 & 0,11 & 0,29 & 2,88 & 136 & - \\
\hline UWO 12 & 2922 & 35 & 235 & 550 & 58 & 78 & 100 & 30 & -206 & $162-165$ & " & 36 & 19 & 30 & 2 & 13 & 0,35 & 0,83 & 0,52 & 0,07 & 0,13 & 13,67 & 127 & 255 \\
\hline UWO 34 & 2921 & 35 & 112 & 290 & 58 & & 090 & 25 & -154 & $108-114$ & $"$ & 46 & 17 & 20 & 9 & 8 & 0,18 & 0,45 & 0,36 & 0,19 & 0,53 & 2,33 & 306 & 8 \\
\hline & " & & $"$ & & & & & $"$ & $"$ & $114-120$ & $"$ & 49 & 17 & 18 & 6 & 11 & 0,21 & 0,37 & 0,34 & 0,12 & 0,35 & 3,17 & 400 & 10 \\
\hline UWO 37 & $"$ & 35 & 18 & 860 & 58 & 803 & 360 & 23 & -228 & $249-252$ & " & 41 & 20 & 16 & 16 & 8 & 0,19 & 0,40 & 0,48 & 0,38 & 0,80 & 1,05 & 528 & 7 \\
\hline UWO 47 & 2822 & 35 & 253 & 360 & 58 & 95 & 900 & 32 & -237 & $249-252$ & " & 34 & 26 & 30 & 9 & 1 & 0,04 & 0,86 & 0,74 & 0,26 & 0,36 & $\mid 1,17$ & 406 & 5 \\
\hline UWO 57 & 2823 & 35 & 43 & 900 & 58 & 956 & 670 & 39 & -153 & $144-147$ & $"$ & 35 & 25 & 18 & 12 & 10 & 0,29 & 0,50 & 0,71 & 0,35 & 0,50 & 1,42 & 485 & 200 \\
\hline & " & & " & & & & & & & 192-195 & $"$ & 45 & 23 & 20 & 12 & 2 & 0,04 & 0,42 & 0,51 & 0,27 & 0,53 & 1,58 & 402 & 86 \\
\hline UWO 76 & 2820 & 35 & 015 & 560 & 58 & 902 & 210 & 51 & -189 & $181-186$ & " & 39 & 28 & 23 & 7 & 5 & 0,12 & 0,58 & 0,71 & 0,18 & 0,25 & $3,21 \mid$ & 400 & 138 \\
\hline UWO 88 & 2620 & 35 & 03 & 480 & 59 & $17 \varepsilon$ & 850 & 10 & -198 & $156-160$ & $"$ & 23 & 16 & 39 & 22 & - & - & 1,51 & 0,70 & 0,96 & 1,35 & 1,04 & 105 & - \\
\hline Uwo 92 & 2519 & 34 & 892 & 260 & 59 & 272 & 250 & 13 & -217 & $77-80$ & $? \mathrm{qe} / / \mathrm{gf}$ & 47 & 16 & 14 & 22 & 1 & 0,03 & 0,31 & 0,35 & 0,46 & 1,32 & 0,67 & 413 & - \\
\hline & & & & & & & & & & $173-180$ & $\mathrm{ge} / \mathrm{Mo}$ & 23 & 13 & 40 & 7 & 18 & 0,77 & 1,72 & 0,55 & 0,30 & 0,55 & $5,67 \mid$ & 601 & 2 \\
\hline UW0115 & 2418 & 34 & 82 & 530 & 59 & 36 & 980 & 1 & $-151 ?$ & $97-104$ & $\mathrm{qe} / / \mathrm{gf}$ & 51 & 28 & 12 & 1 & 9 & 0,17 & 0,24 & 0,55 & 0,01 & 0,01 & 41 & 328 & - \\
\hline Br. I & 3022 & 35 & 267 & 760 & 58 & 67 & 200 & 31 & -81 & $16-18$ & $\mathrm{qD} / / \mathrm{gf}$ & 52 & 24 & 16 & - & 8 & 0,15 & 0,30 & 0,47 & - & - & - & 529 & 20 \\
\hline & " & & & & & 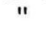 & & & & $111-112$ & $\mathrm{qe} / / \mathrm{gf}$ & 45 & 29 & 8 & 9 & 9 & 0,20 & 0,18 & 0,64 & 0,19 & 0,19 & 0,97 & 457 & 19 \\
\hline NA 99 & "I & 35 & 31 & 490 & 58 & 66 & 870 & 44 & -76 & $72-78$ & $"$ & 47 & 23 & 25 & - & 5 & 0,10 & 0,53 & 0,48 & - & - & - & 211 & - \\
\hline " & $"$ & & & & & & & & & $114-120$ & " & 48 & 37 & 10 & 1 & 4 & 0,08 & 0,2 & 0,75 & 0,02 & 0,02 & 10 & 389 & 2 \\
\hline NA 115 & 3126 & 35 & 73 & 740 & 58 & 55 & 445 & 49 & \pm 0 & $48-51$ & " & 40 & 21 & 19 & 12 & 8 & 0,20 & 0,48 & 0,53 & 0,30 & 0,30 & 1,59 & 352 & - \\
\hline NA 120 & 3127 & 35 & 81 & 840 & 58 & 54 & 480 & 79 & $=86$ & 132 & " & 32 & 8 & 37 & 23 & - & - & 1,14 & 0,26 & 0,71 & 0,71 & 1,60 & 109 & - \\
\hline $\mathrm{NA} 131$ & 3126 & 35 & 717 & 720 & 58 & 57 & 760 & 74 & -116 & $177-183$ & " & 43 & 18 & 29 & 8 & 2 & 0,04 & 0,68 & 0,41 & 0,19 & 0,19 & 3,48 & 364 & 13 \\
\hline NA 144 & 3330 & 44 & 12 & 820 & 58 & 33 & 825 & 86 & -72 & $54-57$ & ?qe//gf & 47 & 11 & 16 & 14 & 13 & 0,26 & 0,33 & 0,23 & 0,30 & 0,30 & 1,11 & 64 & 2 \\
\hline & & & & & & & & . & & $\mid 156-158$ & $\mathrm{qe} / / \mathrm{gf}$ & 33 & 22 & 17 & 15 & 13 & $\mid 0,39$ & $0,53 \mid$ & $|0,67|$ & 0,46 & 0,46 & 1,15 & 447 & 8 \\
\hline
\end{tabular}

Tabelle 2: Kiesanalysen der Fraktion 4-6,3 mm aus Bohrungen im nordöstlichen Niedersachsen in \%; dazu Lokalgeschiebe (zumeist Tertiär) in Stückzahlen, in Ue 1333 Lydite*). $\mathrm{K}=$ nordisches Kristallin; $\mathrm{S}=$ nord. Sediment (Quarzit, Sandstein, Tonstein); $\mathrm{PK}=$ nord. paläoz. Kalkstein; $\mathrm{F}=$ Flint; $\mathrm{Q}=$ Quarz; $\mathrm{GO}=$ Geländeoberfläche; $\mathrm{Q}-\mathrm{NN}=$ Quartärbasis in $\mathrm{m}$ zu NN. Analysen: K.-D. MeYer u. Abdel Moitr; NA 144: H. H. Voss. 
den Rinnen einen älteren elsterzeitlichen Vorstoß repräsentieren, den er mit der HelmeKaltzeit parallelisieren möchte. Nach W. Richter et al. (1968) ist die „... Sandfolge unterhalb des Lauenburger Tons ausgangs der älteren Elster oder einer präelsterzeitlichen Vereisung (Neetze-Eiszeit) entstanden", wobei die Schüttung der präsaalezeitlichen Sedimente in den Rinnen von $S$ nach $N$ erfolgt sein soll. Läßt sich anhand der Kiesuntersuchungen jeglicher Materialtransport von S verneinen, so liegt für eine „Neetze“-Eiszeit kein einziger Hinweis vor. Unterhalb der holsteinzeitlichen Serie lassen sich weder interglaziale noch interstadiale Bildungen nachweisen. Das gilt auch für die „Billbrook“-Interstadiale K. RICHTER's (1962). Bei den kalkfreien Sedimenten, mit denen interstadiale Entkalkungsphasen bezeugt werden sollen, dürte es sich um schon primär kalkarme bis kalkfreie umgelagerte tertiäre Sande handeln, die durch saure Tiefenwässer ihren Karbonatgehalt völlig verloren haben. Eine subaerische interstadiale Entkalkung wäre weiterhin nur möglich gewesen, wenn die in den Rinnen in mehreren 100 m Tiefe liegenden Sedimente längere Zeit canonartig zutage gelegen hätten, was morphologisch wie hydrographisch auszuschließen ist. Alles spricht hingegen dafür, daß der Zeitraum zwischen dem Abschmelzen des Elster-Eises (mit Ausnahme des versenkten Toteises) und der Sedimentation des Lauenburger Tones relativ gering war. Selbst in den Regionen, in denen eine Aufspaltung der

Elster in 2 Phasen nachgewiesen ist wie im Leipziger Raum, finden sich zwischen den beiden elsterzeitlichen Grundmoränen keinerlei Hinweise für interstadiale Bodenbildungen oder Entkalkungsphasen, so daß von Eissmann (1975) lediglich ein kurzes („Miltitzer“) Intervall angenommen wird. U. a. aus diesem Grunde ist eine Parallelisierung der älteren Elster-Moräne mit den Schottern der prä-cromerzeitlichen Helme-Kaltzeit nicht möglich.

Gibt es somit keinerlei Beweise für ein älteres als elsterzeitliches Alter auch der tieferen Rinnen-Sedimente, so läßt sich das elsterzeitliche Alter durch Geschiebezählungen wahrscheinlich machen. Proben aus Bohrungen zeigten das gleiche Geschiebespektrum wie aus oberflächennahen elsterzeitlichen Sedimenten entnommene Proben (MEYER 1970). Diese Geschiebespektren zeichnen sich gegenüber den saalezeitlichen durch einen merklich höheren Anteil norwegischer Leitgeschiebe aus (hauptsächlich Rhombenporphyre), wogegen die ostbaltischen Geschiebe (Åland-Gesteine, Ostsee-Porphyre) stark zurücktreten oder sogar fehlen können. Nach E zu schwächt sich diese Tendenz ab, aber auch noch die Proben im Lüneburger Raum zeigen diesen starken Gehalt an Oslo-Geschieben, z. B. in Bohrungen des WW Adendorf. Die auf Tab. 1 wiedergegebenen Zählungen entstammen unmittelbar benachbarten Bohrungen. Die TGZ dieser 5 Proben weisen einheitlich die geschilderte Tendenz auf. Bemerkenswert ist, daß unter den 12246 Geschieben nur ein einziger Lydit südlicher Herkunft ist; auch unter dem feineren Material befand sich nur einer. Solche vereinzelten Kieselschiefer dürften in erster Linie mehrfach umgelagertem „präglazialen“ Material entstammen (Meyer in Duphorn et al. 1975); im östlichen Niedersachsen kommen dafür auch die Loosener Kiese infrage. Im Raum Lüchow-Dannenberg sind Kieselschiefer nach neueren Untersuchungen bereits in elsterzeitlichen Schmelzwassersedimenten verbreitet. Wie bereits früher dargelegt (LüTTIG \& MEYER 1974), haben die Kieselschiefer mit den Rinnen nichts zu tun.

\title{
4. Entstehung der Rinnen
}

Für die Entstehung der Rinnen werden heute von den verschiedenen Autoren im wesentlichen 5 Ursachen, teils allein, teils in Kombination, herangezogen:

\author{
Subrosion oder/und Halokinese \\ Tektonik \\ Fluviatile Erosion \\ Glaziale Erosion \\ Glazifluviatile (subglaziale) Erosion.
}


In mehreren Arbeiten haben JoHANnSEn (1960, 1964, 1971), JOHANNSEN \& LöHNERT (1971, 1974), Johannsen \& Nachtigall (1972), ferner LöHneRt (1966) die schleswigholsteinischen Rinnen mit salinartektonischen Einflüssen in Zusammenhang gebracht. Nach W. Schulz (1967) sind in SW-Mecklenburg lokale Mächtigkeiten des Quartär von 300 bis $400 \mathrm{~m} \mathrm{z}$. T. in den Randsenken, z. T. auch auf dem Top halokinetischer Strukturen erbohrt. Ruppert \& SCHNEIDER (1975) deuten die Becken, in denen der Lauenburger Ton abgesetzt wurde, salinartektonisch, diejenigen über den Salzstrukturen als durch Suberosion entstanden. Auf unsere eigenen Beobachtungen im Zusammenhang mit Salzstrukturen haben wir bereits hingewiesen. Wenn auch deren Einfluß auf die Rinnen kaum zu leugnen ist, kann jedoch von einer generellen Abhängigkeit der Rinnen von ihnen nicht die Rede sein.

Daß die großen Quartärmächtigkeiten im Hamburger Raum nicht tektonisch bedingt sind, hatte bereits Wolff erkannt. Auch an anderen Stellen fanden sich dafür keine Beweise. Dies sei deshalb betont, weil neuerdings (Paluska, Vortrag Symposium Rosenheim) wieder derartige Ansichten vorgetragen werden und auch im Deutschen Planungsatlas Band Hamburg (1978) kartenmäßig dargestellt sind. Dabei werden auch jungquartäre kräftige tektonische Bewegungen angenommen. PALuskA (1976) gibt dabei z. B. an: „Die Sedimente des Lauenburger-Holstein-Seesystems sind jedoch örtlich durch nacheemzeitliche tektonische Vorgänge bis auf $+40 \mathrm{~m} \mathrm{NN}$ (Lauenburg) hochgehoben worden." Nun ist seit langem bekannt und auch im Gelände jederzeit nachprüfbar, daß bei Lauenburg eindeutig Eisstauchungen vorliegen, wobei die Stauchungen teilweise einzelnen Phasen zugeordnet werden können (durch diskordante Überlagerungen der Moränen über dem jeweiligen gestauchten Untergrund; MEYER 1965). Auch müßte der heute durchschnittlich bei $20 \mathrm{~m}$ über NN liegende Eem-Torf bei $-20 \mathrm{~m}$ NN entstanden sein. Die Umdeutung (ohne jeden Beweis!) dieser eindeutigen Befunde zugunsten tektonischer Hypothesen kann daher nur als Spekulation bezeichnet werden, zumal wenn auch so hervorragend bekannte Gebiete wie dasjenige zwischen Saale und Elbe, welches sich nach EIsSmanN (1975) während des gesamten Känozoikums als relativ stabil erwiesen hat, entgegen den Tatsachen in die "Beweisführung“ einbezogen wird. Jedenfalls haben die anderenorts nachgewiesenen quartären Bewegungen offenkundlich mit der Genese der Rinnen nichts zu tun.

Die Entstehung der Rinnen durch fluviatile Erosion ist in den letzten Jahren erneut diskutiert worden. Das setzt jedoch eine Hebung des norddeutschen Flachlandes (und angrenzender Gebiete) um ca. $400 \mathrm{~m}$ voraus, was u. a. von CePEK $(1967,1968)$ auch angenommen wird.

Nun wäre eine post-pliozäne Hebung und präelsterzeitliche Senkung um diesen Betrag für unser Gebiet von einer im Vergleich zu den epirogenetischen Vorgängen des Neogens geradezu gewaltigen Größenordnung, für die es keine Beweise gibt. Bei einem derartigen mittelgebirgsähnlichen Relief (in diesen Lockersedimenten ohnehin nicht möglich) müßten große Mengen fluviatiler Schotter in den Tälern weit nach $\mathrm{N}$ transportiert worden sein, zumindest in den präelsterzeitlichen Kaltzeiten. Wie geschildert, kennt man keine derartigen tiefliegenden Sedimente. Auch die Annahme einer späteren totalen Ausräumung ist wenig wahrscheinlich, zumal man dann wenigstens die umgelagerten Kiese finden müßte.

Statt in den Rinnen finden sich jedoch die „präglazialen“ Schotter sowohl in den südlichen Randgebieten (EIsSMANN 1967, 1975) wie in SW-Mecklenburg (voN BüLOw 1967) a u f den Hochflächen, die ihrerseits von den jüngeren Rinnen zerschnitten werden. (Gleiches ist offensichtlich in S-Polen der Fall; für die dort postulierten „cromerzeitlichen fluviatilen Rinnen" ist das cromerzeitliche Alter sowie die fluviatile Genese zumindest fraglich. 
Auch im Allergebiet zwischen Celle und Gifhorn ist aus Teufen wenig unter NN in Bohrungen ein das Tertiär überlagernder altquartärer Schotterkörper bekannt, der überwiegend aus Quarz und einheimischen Gesteinen aufgebaut ist und anscheinend kein oder nur Spuren nordischen Materials enthält. Eine Kiesprobe (Tab. 3) aus der Bohrung NA 141 Oerreler Heide, entnommen bei $84-87 \mathrm{~m}$ u. Gelände ( -7 bis $-10 \mathrm{~m} \mathrm{NN}$ ) enthielt kaum Flint; letzterer dürfte ebenso wie der geringe nordische Kristallin-Anteil Nachfall aus den hangenden rein nordischen Sedimenten sein. Die große Menge von Quarzit und Restquarz könnte umgelagerten tertiären Sedimenten entstammen. Die übrigen Komponenten, vor allem Gangquarz, Lydit und südliche Porphyre, beweisen einen Transport aus südlichen Richtungen. Um welches Flußsystem es sich handelt, könnte eine genauere Analyse der Porphyre ergeben. Dies setzt jedoch ein reicheres Material voraus. Ob Beziehungen zu den von Genieser (1970) erwähnten Kiesen von Gr. Hehlen bei Celle bestehen, ist noch offen. Da die Schotter von der Elster-Moräne überlagert werden, können sie einer früh-elsterzeitlichen Oberterrasse entsprechen. Jedenfalls würde man bei präcromerzeitlichen Kiesen eine stärkere Verwitterung namentlich der Porphyre erwarten dürfen.

Wichtiger als die genaue Einstufung ist die Tatsache, daß auch in diesem Raum Rinnen existieren, die den Schotterkörper durchschneiden und die wiederum praktisch ausschließlich nordisches Material führen (als Beispiel sei auf die Bohrung NA 144, Malloh, $20 \mathrm{~km}$ NE Gifhorn, Tab. 2, verwiesen). Letzteres gilt auch für alle Rinnen nördlich der „Maarleveld'schen Linie“, der Verbreitungsgrenze der alten Weserkiese (MAAR-

\begin{tabular}{|c|c|c|}
\hline Stückzahlen in $:$ & $4-6,3 \mathrm{~mm}$ & $6,3-12,5 \mathrm{~mm}$ \\
\hline \multicolumn{3}{|l|}{ Nordische Komponente } \\
\hline Kristallin & 2 & 4 \\
\hline F1int & 1 & - \\
\hline \multicolumn{3}{|l|}{ Tertiäre_(?2_Komponente } \\
\hline Quarzit & 20 & 27 \\
\hline \multicolumn{3}{|c|}{ Einheimisch-mesozoische_Komponente } \\
\hline Sandstein & 6 & 7 \\
\hline Hornstein & 1 & 3 \\
\hline \multicolumn{3}{|c|}{ Einheim.-paläozoische Komponente } \\
\hline Lydit & 1 & 3 \\
\hline Porphyr & 3 & 7 \\
\hline Sediment & 3 & 5 \\
\hline \multicolumn{3}{|l|}{ Quararz } \\
\hline Restquarz & 31 & 7 \\
\hline Milchquarz (Gangqu.) & 34 & 37 \\
\hline Anzahl der Gerölle & 612 & 137 \\
\hline
\end{tabular}

Tab. 3: Kiesanalysen einer vermutlich frühelsterzeitlichen Oberterrasse aus $84-86,5 \mathrm{~m}$ Tiefe. Bohrung NA $14117 \mathrm{~km} \mathrm{~N}$ Gifhorn, TK 25 Wahrenholz Nr. 3329, R 4302630 H 5834730 , Ansatzpunkt 77,5 m + NN. Analyse: K.-D. Meyer. 
leveld 1954). Die Aussage von Ortlam \& Vierhuff (Manuskript 1978), daß sich das Mittelgebirgsmaterial überwiegend in den südlichen Abschnitten der Rinnensysteme ablagerte, ist nicht belegt. Auch die alte, von Grimmel (1973) wieder aufgegriffene Ansicht, Schmelzwässer und Mittelgebirgsflüsse seien subglaziär nach NW bis $\mathrm{N}$ geflossen, läßt sich durch keinen Beweis stützen. Zu Recht hat WoldstedT diese „erweiterten Perspektiven" (GRIMmel 1973: 81) $\mathrm{n} \mathrm{i} \mathrm{cht} \mathrm{in} \mathrm{die} \mathrm{"herrschende} \mathrm{Lehrmeinung} \mathrm{"übernommen.}$ Während sich die alten Weserkiese bekanntlich sehr gut nach W bis in die Niederlande verfolgen lassen, fehlen Weserspuren $\mathrm{N}$ der "Maarleveld'schen Linie“ gänzlich. Der Durchbruch der Weser durch die Nienburger Geest erfolgte erst nach der Hamelner Phase des Drenthe-Stadiums. Vorher ging der Abfluß immer nach W. Ein Abdrängen durch die Gletscher ist nicht nachweisbar. Selbst nach Ende der Elster-Vereisung gelang es der Weser nicht, über Nienburg in die wenig weiter $\mathrm{N}$ einsetzende tiefe Verdener Rinne auf Blatt 3221 Eystrup „überzulaufen“, die sicherlich im Holstein-Interglazial noch morphologisch

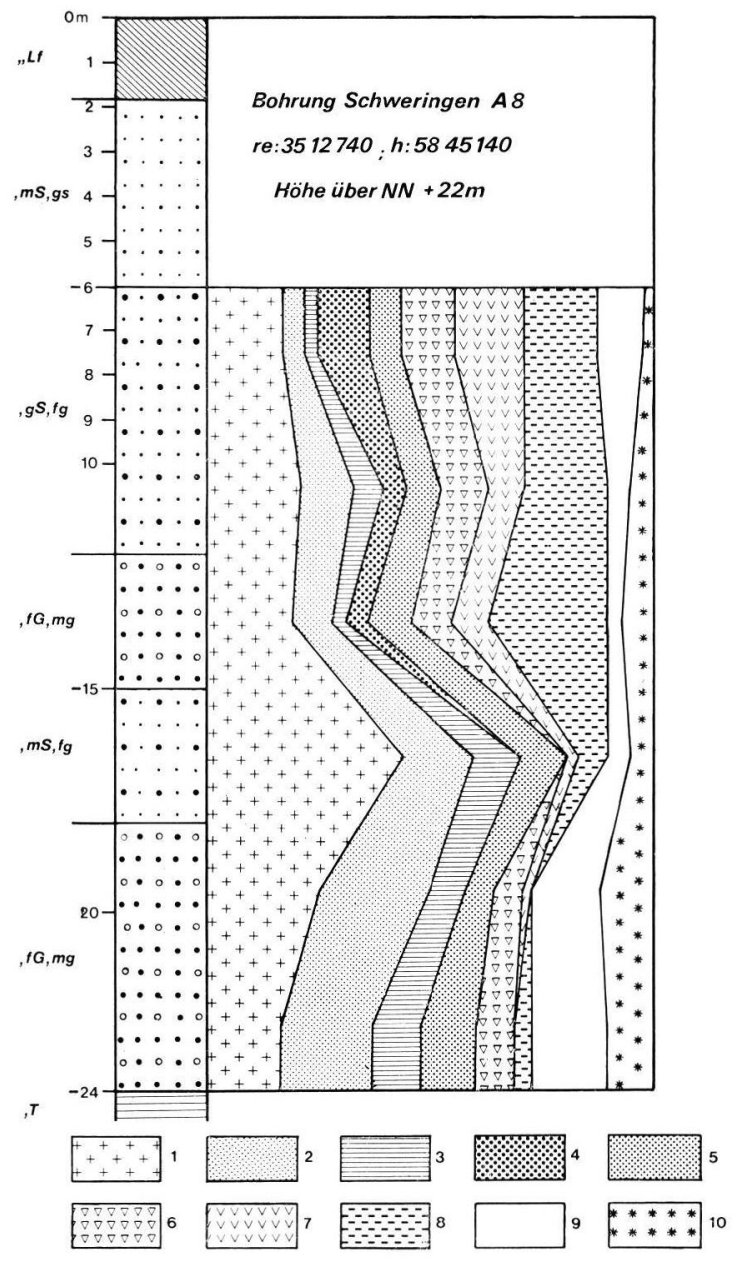

Abb. 5: Feinkiesanalysen aus der Bohrung Schweringen A 8, Fraktion 4-6,3 mm. TK 25 Eystrup Nr. 3221. $1=$ Nordisches Kristallin; $2=$ Nordisches Sediment; $3=$ Flint; $4=$ Buntsandstein; $5=$ übriges einheimisch-mesozoisches Sediment; $6=$ Lydit; $7=$ Thüringer Wald-Porphyr, $8=$ übriges einheimisch-paläozoisches Sediment; $9=$ Restquarz; $10=$ Milchquarz. 
wirksam war. Dies geht u. a. aus Bohrungen bei Schweringen (B1. Eystrup) hervor, in denen unter dem Kieskörper der Niederterrasse nur noch nordisches Material existiert, obgleich die Quartärbasis bis $-113 \mathrm{~m} \mathrm{NN}$ abfällt. Abb. 5 zeigt die Zusammensetzung der groben Feinkiesfraktion (4-6,3 mm) des Teufenbereichs 6-24 $\mathrm{m}$ der Bohrung A 8. Deutlich wird dabei eine petrographische Zweiteilung in eine obere Einheit $(6-15 \mathrm{~m}) \mathrm{mit}$ niedrigen, sowie eine untere Einheit (15-24 m) mit doppelt so hohen Anteilen nordischen Materials. Diese auch von Plischke (1976) auf Blatt 3420 Stolzenau sowie in Kartierbohrungen auf Blatt 3320 Liebenau von K.-D. MEYER 1977 gefundene untere Einheit entspricht wahrscheinlich einer spätsaalezeitlichen (Jüngere Drenthe bis Warthe) Weser-Terrasse. Die Quartärfolge weiter unterhalb enthielt in dieser sowie in drei weiteren Bohrungen kein südliches Material mehr, obgleich das nächstgelegene Vorkommen von Mittelterrassenkiesen nur $5 \mathrm{~km}$ weiter SW liegt.

Daß die späteren Flüsse den alten Rinnen im allgemeinen nicht folgen können, liegt neben den lokalen Untergrundsverhältnissen (sperrende Präquartär-Riegel) in erster Linie wohl daran, daß die meisten Rinnen nach unserer heutigen Kenntnis mehr oder weniger abrupt enden, ohne den Fuß der Mittelgebirge zu erreichen. Sie haben offenkundlich mit einem fluviatilen Entwässerungsnetz nichts $\mathrm{zu}$ tun (fluviatile Einschneidungen im Bergland bleiben davon unberührt). Daß Verlauf, Anzahl, Querschnitt etc. der Rinnen einem fossilen Flußnetz ohnehin wenig ähneln, sei nur am Rande vermerkt; WoLDSTEDT \& DUPHORN (1974) haben auch darauf hingewiesen.

Nach den vorliegenden Fakten verbleiben damit für die Entstehung der Rinnen die Möglichkeiten der glazialen bzw. glazifluviatilen Erosion. Für beide Ansichten finden sich Vertreter. In Hamburg hat КосH (1924) die „Becken“ als durch Gletscherzungen entstanden erklärt. GRIPP (1964) modifizierte diese Vorstellung. Er nimmt an, . . „ daß Inlandeis und Großgletscher kleine Eiszungen aussandten, die teils infolge stärkeren Gefälles, teils durch längere Dauer tief exarierten. Dies geschah zu verschiedenen Zeiten. Entsprechend konnten Schurfrinnen neben- und übereinander entstehen. Scheinbar zusammengehörige Hohlformen können also ohne jeden zeitlichen Zusammenhang sein".

Sicherlich gibt dieses Modell eine Erklärung für spezielle Fälle, es entspricht jedoch nicht dem in Niedersachsen entwickelten Normaltyp. Das bei GripP auf S. 201 abgebildete Rinnenschema mit mächtiger Grundmoräne an der Basis ist äußerst selten entwickelt. Auch in Hamburg (GRUBE \& EHLERs 1975) „... überwiegt bei den tieferen elsterzeitlichen Sedimenten das Glazifluvium und bezeugt die subglaziale Tätigkeit der Schmelzwässer“. Nach LöHNERT (1966) gehen blind endende Abzweigungen auf Schmelzwassererosion zurück.

Da bei der überwiegenden Zahl der niedersächsischen Rinnen Grundmoräne fehlt oder nur in den oberen Abschnitten auftritt, kann Gletschererosion nicht die Hauptursache gewesen sein. Auch kann ehedem vorhandener mächtiger Geschiebemergel nicht in großem Stil zerstört worden sein, denn wie in den Zungenbecken wäre er in den tiefen Rinnen gut geschützt; zudem hätte eine Auswaschung viel mehr grobes Material hinterlassen. Es wird daher angenommen, daß unsere Rinnen in erster Linie durch die Tätigkeit subglazialer Schmelzwässer ausgekolkt wurden, wobei das Ausmaß vorhergehender oder in Teilbereichen gleichzeitiger Gletschererosion schwer abzuschätzen ist. Letzteres scheint bei flacheren, aber breiteren Rinnen stärker der Fall zu sein. Aber auch hier liegt die Grundmoräne häufig nicht direkt dem Untergrund auf.

Die Theorie der Entstehung durch subglaziale Schmelzwasser-Erosion wirft einige Fragen auf. Schwer abschätzbar sind die hydraulischen Bedingungen des angenommenen „Eis-Karstsystems“. Eine andere, häufig gestellte Frage nach dem Verbleib des „ausgespülten" Materials ist beantwortbar: Es wurde in die normalen Verschüttsande eingearbei- 
tet. Große Mengen des Quarzes derselben entstammen tertiären Sedimenten. Auch der Glaukonit, der namentlich im SW des Gebietes einen höheren Prozentsatz der Schmelzwassersande bildet, ist gleicher Herkunft. Da hier - etwa W der Hunte - normalerweise die glaziären Sedimente vom glaukonitführenden Tertiär durch mächtige glaukonitfreie pliozäne Quarzsande getrennt sind, kann der Glaukonit im Quartär nur aus Zonen tieferer Erosion stammen; die wenigen tertiären Aufragungen sind dafür allein nicht ausreichend. Schließlich ist im weiteren Verlauf der Vereisung ein Teil des ausgespülten Materials vom Gletscher verfrachtet und inkorporiert worden. Regionale Untersuchungen der Leichtminerale, u. a. des Glaukonits, könnten hier interessante Hinweise geben.

Es ist allerdings schwierig zu erklären, warum offenbar nur während der Elster-Eiszeit derartig tiefe Rinnen entstanden. Dies könnte mit der Art der Gletscherbewegung (LouIs 1952; Pillewitzer 1969) oder auch mit der elsterzeitlichen Eisbewegungsrichtung zusammenhängen (in Niedersachsen $\mathrm{N}-\mathrm{S}$ ). Möglich sind auch klimatische Ursachen; vielleicht war der Schmelzwasseranfall während der Elster besonders hoch - ein Gedanke, den wohl zuerst Eissmann geäußert hat. Das von Eissmann (1967, 1975) gegebene Modell der "glazihydromechanischen Strukturen" ist auch für die niedersächsischen Verhältnisse gut anwendbar. Mit den von voN BüLOw (1967) aus Mecklenburg beschriebenen Rinnen und deren Deutung durch subglaziäre Erosion besteht ebenfalls weitgehende Übereinstimmung.

Ohne damit verallgemeinern zu wollen, stellt sich die Frage, ob ein Teil der mit mächtigem Lauenburger Ton gefüllten Depressionen in den Niederlanden (Zonneveld 1958; Ter Wee 1952; De Jong 1965) nicht ebenfalls durch subglaziale Erosion gebildet wurde. Bisher sind jedoch in den Niederlanden wie auch in Niedersachsen westlich der Ems keine Elster-Grundmoränen, sondern nur elsterzeitliche Schmelzwassersedimente bekannt.

\section{Bemerkungen zur Karte der Quartärbasis}

Die vorliegende „Karte der Quartärbasis von Mittel- und Nordost-Niedersachsen“ wurde, teilweise von Arbeitskarten 1:25000 ausgehend, im Maßstab 1:200 000 erarbeitet. Sie konnte aus drucktechnischen und finanziellen Gründen leider nur in stark verkleinertem Maßstab veröffentlicht werden. Dies ist um so bedauerlicher, als inzwischen für Schleswig-Holstein eine von Hinsch (1977) veröffentlichte farbige Karte im Maßstab $1: 250000$ vorliegt und ein direkter Anschluß wünschenswert gewesen wäre. Der Kartenausschnitt wurde so gewählt, um einerseits das durch die Bohrungen der wasserwirtschaftlichen Rahmenplanung bis jetzt besonders gut erkundete Unterelbe-Unterweser-Gebiet einzubeziehen, andererseits aber auch den Anschluß an den Rand des niedersächsischen Berglandes mit dem Austritt der Flüsse zu erreichen, um mögliche Beziehungen derselben $\mathrm{zu}$ den Rinnen zu untersuchen.

Insgesamt sind für die Karte einige 10000 Bohrprofile durchgesehen worden. Die Qualität der Bohrprofile schwankt jedoch sehr. So erreichen manche neuere Beschreibungen häufig nicht die Qualität älterer Aufnahmen, was keineswegs allein an dem heute allgemein verwendeten Druckspülbohrverfahren liegt. Auch Bohrlochmessungen können gute petrographische und genetische Angaben nicht ersetzen. Besonders schwierig ist es, pleistozäne von darunterliegenden tertiären Sanden zu trennen (voN BüLow 1967: 406). Erdölbohrungen, bei denen in der Regel wenig Wert auf die Beschreibung des Quartärs gelegt wird, sind dennoch besonders im S, wo pleistozäne Sedimente unmittelbar auf alttertiären oder noch älteren liegen, häufig eine wichtige Hilfe. Obwohl einige der dargestellten Bohrungen die Quartärbasis nicht erreicht haben, waren sie trotzdem wichtig für die Ermittlung der Mindestteufen von Rinnen. 
Auswertbar für die Konstruktion der Quartärbasis erschienen rund 15000 Bohrprofile. Von diesen ist wieder nur ein geringerer Teil in die Karte übernommen worden, da sich die Bohrungen stellenweise zu sehr häufen. In dem bearbeiteten Bereich ist der Kenntnisstand jedoch nicht einheitlich. Die Zahl der auswertbaren Bohrungen schwankt zwischen 20 und über 200 pro Blatt der TK 25. So ist auf manchen Blättern eine Konstruktion der Quartärbasis im 10-m-Abstand möglich, wovon bei der Generalisierung auf 1:200000 schon viele Details verloren gingen. Besonders in Gebieten, die nur durch relativ wenige Bohrungen erschlossen sind, war die Geoelektrik z. T. sehr hilfreich. Dagegen sind z. B. im Küstenbereich, wo Grundwasser oberflächennah versalzt ist - und daher von der Geoelektrik keine brauchbaren Ergebnisse zu erwarten sind -, bei einem Bohrungsabstand von einigen $\mathrm{km} \mathrm{z}$. T. bei der Rinnenkonstruktion mehrere Varianten denkbar. Bei jeder Darstellung sind aber solche Unsicherheiten in Kauf zu nehmen; schließlich ist die Karte auch als Arbeitskarte gedacht, um die einzelnen Strukturen zu verfolgen und mit Hilfe neuerer Bohrungen ein verbessertes Bild geben zu können. Eine detaillierte Beschreibung der einzelnen Rinnen setzt weitere Bohrungen voraus, desgl. weitere lithologische Untersuchungen.

Auch eine morphogenetische Differenzierung der Quartärbasis, wie sie WorTMANN (1968) im Wiehengebirgsvorland gelang, erfordert ein erheblich dichteres Netz an lithologisch bearbeiteten Bohrungen.

\section{Schriftenverzeichnis}

Bankwitz, P. (1971): Bemerkungen zur Karte der Tiefenlage der Quartärbasis in der DDR. Petermanns Geogr. Mitt., 115 (2): 141-143, 1 Kt.; Gotha/Leipzig.

Benda, L. \& Brandes, H. (1974): Die Kieselgur-Lagerstätten Niedersachsens. T. Verbreitung, Alter und Genese. - Geol. Jb., A 21: 3-85, 9 Abb., 3 Tab., 7 Taf.; Hannover.

BüLow, W. v. (1967): Zur Quartärbasis in Mecklenburg. — Ber. deutsch. Ges. geol. Wiss. A. Geol. Paläont., 12 (3/4): 405-413, 3 Abb.; Berlin.

- (1969): Altpleistozäne Schotter (Loosener Kiese) in Südwestmecklenburg mit nordischen und südlichen Geröllen. - Geologie, 18 (5): 563-589, 7 Abb., 4 Taf., 3 Tab.; Berlin.

CepeK, A. G. (1967): Stand und Probleme der Quartärstratigraphie im Nordteil der DDR. Ber. deutsch. Ges. geol. Wiss. A. Geol. Paläont., 12 (3/4): 375-404, 4 Abb., 1 Tab.; Berlin.

- (1968): Quartär. - in: Grundriß der Geologie der Deutschen Demokratischen Republik: 385-425, 2 Abb., 1 Tab.; Berlin.

Duphorn, K., Grube, F., Meyer, K.-D., Streif, H. \& Vinken, R. (1973): State of Research on the Quaternary of the Federal Republik of Germany. A. Area of Scandinavian Glaciation. 1. Pleistocene and Holocene. - Eiszeitalter u. Gegenwart, 23/24: 220—250, 1 Abb.; Öhringen/Württ.

Eissmann, L. (1967): Glaziäre Destruktionszonen (Rinnen, Becken) im Altmoränengebiet des Norddeutschen Tieflandes. - Geologie, 16: 804-833, 19 Abb., 1 Tab.; Berlin.

- (1975): Das Quartär der Leipziger Tieflandsbucht und angrenzender Gebiete um Saale und Elbe. - Schriftenr. f. geol. Wiss., 2: 228 S., 58 Abb., 23 Tab., 17 Taf.; Berlin.

Genieser, K. (1970): Über Quarze, Amethyste und verkieselte Fossilien. - Grondboor en hamer, 1970 (2): 35-64, 4 Abb., 3 Tab.; Enschede.

Gotтsche, C. (1897): Die tiefsten Glacialablagerungen der Gegend von Hamburg. - Vorläufige Mitteilung. - Mitt. d. Geogr. Ges. in Hamburg, 13: 131-140; Hamburg.

- (1901): Der Untergrund Hamburgs. - 15 S., 2 Abb., 1 Tab.; Hamburg.

Grahmann, R. (1937): Form und Entwässerung des nordeuropäischen Inlandeises. - Mitt. Ges. Erdkunde Leipzig, 54: 48-70, 3 Abb.; Leipzig.

Gripp, K. (1956): Mindel-Vereisung und stör-zeitlicher Meeresspiegel. - Meyniana, 5: 3-6; Kiel.

- (1964): Erdgeschichte von Schleswig-Holstein. - 411 S., 57 Taf., 3 Kt.; Neumünster.

Groba, E., Ortlam, D. \& Vierhuff, H. (1970): Pleistozäne Rinnen in der Lüneburger Heide. Eiszeitalter u. Gegenwart, 21: 185-186; OOhringen/Württ.

Grube, F. \& Ehlers, J. (1975): Pleistozäne Flußsedimente im Hamburger Raum. - Mitt. Geol.Paläont. Inst. Univ. Hamburg, 44: 353-382, 1 Taf., 6 Abb.; Hamburg. 
Hannemann, M. \& Radtke, H. (1961): Frühglaziale Ausräumungszonen in Südostbrandenburg. Neue Ergebnisse zur Paläogeographie und Stratigraphie des Quartärs. - Z. angew. Geol., 7 (2): 69-74, 2 Abb.; Berlin.

Hannemann, M. (1964): Quartärbasis und älteres Quartär in Ostbrandenburg. - Z. angew. Geol., 10 (7): 370-376, 1 Abb.; Berlin.

Heck, H. L. (1939): Die prädiluviale Grundfläche in Schleswig-Holstein. - Jb. preuß. geol. L.Anst., 59: 83-99, 4 Abb.; Berlin.

- (1963): Zur Unterfläche des Quartärs in Mecklenburg. - Berichte der geol. Ges. DDR, 8 (5/6): 501-515, $1 \mathrm{Kt}$; ; Berlin.

Hinsch, W. (1975): Präquartärer Untergrund und glaziäre Rinnen in Südostholstein. - Mitt. Geol.-Paläont. Inst. Univ. Hamburg, 44: 383-402, 4 Abb., 2 Taf., 2 Tab.; Hamburg.

- (1977): Basis des glaziären Pleistozäns in Schleswig-Holstein. - Kt. 1:250000, Hrsg.: Geol. L.-Amt Kiel.

JARITZ, W. (1973): Zur Entstehung der Salzstrukturen Nordwestdeutschlands. - Geol. Jb., A 10: 77 S., 3 Abb., 1 Tab., 2 Taf.; Hannover.

Johannsen, A. (1960): Ur-Anlagen pleistozäner Förden und Rinnen in Schleswig-Holstein. Geol. Jb., 77: 271-308, 11 Abb.; Hannover.

- (1964): Zur Geologie tertiärer und pleistozäner Wasserleiter in Schleswig-Holstein. - Geol. Jb., 81: 739-770, 17 Abb.; Hannover.

- (1971): Salinartektonische Einflüsse auf Ausbildung und Verbreitung tertiärer und quartärer Sedimente in Ost-Holstein. - Meyniana, 21: 33-39, 6 Abb.; Kiel.

Johannsen, A. \& Löhnert, E. (1971): Geologie und Grundwasservorkommen im schleswig-holsteinischen Unterelbe-Gebiet. - Meyniana, 21: 41-66, 18 Abb.; Kiel.

- (1974): Geologie und Grundwasservorkommen im schleswig-holsteinischen Randgebiet östlich von Hamburg. - Meyniana, 25: 31-53, 14 Abb., 4 Tab.; Kiel.

- \& Nachtigall, K. H. (1972): Geologisch-hydrogeologische Untersuchungen im Südteil des Kreises Herzogtum Lauenburg/Schleswig-Holstein. - Meyniana, 22: 71-84, 12 Abb., 1 Tab.; Kiel.

JoNG, J. DE (1965): Quaternary sedimentation in the Netherlands. - Geol. Soc. Amer. Inc. Spec. Pap., 84, VII. Congr. INQUA, Colorado. in: Int. stud. on Quat.: 95-123; New York.

Jordan, H. \& Voss, H.-H. (1978): Geröllanalytische Gliederung der pleistozänen Kiessande nordwestlich von Hannover. - Eiszeitalter u. Gegenwart, 28: 10—38, 8 Abb., 3 Tab.; OOhringen/ Württ.

Косн, E. (1924): Die prädiluviale Auflagerungsfläche unter Hamburg und Umgebung. - Mitt. Min.-Geol. Inst. Hbg., 6: 31-95, 2 Kt., 5 Prof.; Hamburg.

- (1954): Vom Untergrund Hamburgs. - Mitt. Geol. Staatsinst., 23: 10—17, 8 Abb.; Hamburg.

LÖHNERT, E. (1966): Glaziäre Rinnen im Raum Hamburg und ihre Beziehungen zum präquartären Untergrund. - Abh. u. Verh. Nat. Verein in Hamburg, N. F., 10: 47-51; Hamburg.

Louis, H. (1952): Zur Theorie der Gletschererosion in Tälern. - Eiszeitalter u. Gegenwart, 2: 12-24, 3 Abb.; Ohringen/Württ.

LÜтtiG, G. (1958): Methodische Fragen der Geschiebeforschung. - Geol. Jb., 75: 361-418, 17 Abb., 1 Tab., 3 Taf.; Hannover.

- (1972): Die Bodenschätze des Nordsee-Küstenraumes und ihre Bedeutung für Landesplanung und Raumordnung. - N. Arch. f. Nds., 21: 13-25, 6 Abb.; Göttingen.

- \& Meyer, K.-D. (1974): Geological History of the River Elbe, mainly of its lower course. - Cent. soc. géol. Belgique, 1974, 19 S., 1 Abb., 1 Tab.; Brüssel.

MaArleveld, G. L. (1954): Uber fluviatile Kiese in Nordwestdeutschland. - Eiszeitalter u. Gegenwart, 4/5: 10-17, $2 \mathrm{Abb}$; "O'hringen/Württ.

Meyer, K.-D. (1965): Das Quartärprofil am Steilufer der Elbe bei Lauenburg. - Eiszeitalter u. Gegenwart, 16: 47-60, 3 Abb., 1 Taf.; O'hringen/Württ.

- (1970): Zur Geschiebeführung des Ostfriesisch-Oldenburgischen Geestrückens. - Abh. naturw. Verein Bremen, 37: 227-246, 4 Abb., 1 Tab.; Bremen.

Müller, A. (1973): Beitrag zum Quartär des Elbegebietes zwischen Riesa und Wittenberg unter besonderer Berücksichtigung der Elbtalwanne. - Z. geol. Wiss., 1 (9): 1105-1122, 7 Abb., 2 Tab.; Berlin. 
NiEDERmayer, J. (1965): Gliederung und Ausbildung des Quartärs im Niederelbegebiet. - MaxRichter-Festschr.: 73-82, 1 Abb.; Clausthal-Zellerfeld.

Paluska, A. (1976): Entwässerung des norddeutschen Flachlandes im Pleistozän und ihre Folgen für mutmaßliche Schwankungen des Meeresspiegels. - Eiszeitalter u. Gegenwart, 27: 205; Öhringen/Württ.

Pillewitzer, W. (1969): Die Bewegung der Gletscher und ihre Wirkung auf den Untergrund. Z. Geomorph., Suppl.-Bd., 8: 1-10, 3 Fig., 3 Abb., 1 Taf.; Berlin.

Plischke, J. (1976): Untersuchungen zur Geröll- und Schwermineralführung fluviatiler und glazifluviatiler Pleistozänkiese im Raum Nienburg/Weser. - Unveröff. Dipl.-Arbeit TU Hannover, 43 S., 17 Abb., 184 Tab., 6 Taf.; Hannover.

Richter, K. (1962): Geschiebekundliche Gliederung der Elster-Eiszeit in Niedersachsen. - Mitt. geol. Staatsinst. Hamburg, 31: 309-343, 2 Tab., 4 Abb.; Hamburg.

Richter, W., Preul, F., Dechend, W., Dürbaum, H.-J., Groba, E. \& Herrmann, R. (1968): Ein Rückblick auf 20 Jahre hydrogeologischer Arbeiten des Niedersächsischen Landesamtes für Bodenforschung. - Geol. Jb., 85: 817-840, 4 Abb., 3 Taf.; Hannover.

Ruppert, F.-R. \& Schneider, W. (1975): Hinweise auf Zusammenhänge zwischen der Genese des Lauenburger Tons (Elster-Glazial) und den Salzstrukturen Norddeutschlands aufgrund tonmineralogischer Untersuchungen. - N. Jb. Geol. Paläont. Mh., 1975 (10): 606-627, 12 Abb., 2 Tab.; Stuttgart.

Schulz, W. (1967): Abriß der Quartärstratigraphie Mecklenburgs. - Arch. Freunde Naturgesch. Mecklenb., 13: 99-119, 1 Abb.; Rostock.

Ter Wee, M. W. (1962): The Saalian glaciation in the Netherlands. - Meded. Geol. Sticht., 15: 57-74, 16 Fig., 1 Kt.; Maastricht.

WolfF, W. (1907): Der geologische Bau der Bremer Gegend. - Abh. Naturw. Ver. Bremen 1907, 19: 207-216; Bremen.

- (1909): Der Untergrund von Bremen. - Z. dt. geol. Ges., 61, Mbr.: 348-365; Berlin.

- (1913): Erläuterungen zu Bl. Wedel d. Geol. Kt. Preußen 1:25 000, 43 S., 1 Kt., 1 Abb.; Berlin.

- (1914): Erläuterungen zu Bl. Hamburg d. Geol. Kt. Preußen 1:25 000, 58 S.; Berlin.

- (1917): Das Diluvium der Gegend von Hamburg. - Jb. preuß. geol. L.-Anst., 36 (T. II): 227-324, 1 Abb., 5 Taf.; Berlin.

Wortmann, H. (1968): Die morphogenetische Gliederung der Quartärbasis des Wiehengebirgsvorlandes in Nordwestdeutschland. - Eiszeitalter u. Gegenwart, 19: 227-239, 4 Abb., 1 Tab., 1 Taf.; OOhringen/Württ.

Woldstedt, P. \& Duphorn, K. (1974): Norddeutschland und angrenzende Gebiete im Eiszeitalter. - 3. Auflage, 500 S., 91 Abb., 26 Tab.; Stuttgart.

Zonneveld, J. I. S. (1958): Litho-stratigraphische eenheden in het Nederlandse Pleistoceen. Meded. Geol. Sticht., 12: 31-64; Maastricht.

Unveröffentlichte Berichte im Niedersächsischen Landesamt für Bodenforschung, Hannover:

Anrich, H. \& Hofmann, W. (1974): Tätigkeitsbericht über Teile des Raumes „Nördlich der Aller“ der Wasserwirtschaftlichen Rahmenplanung.

BüchNeR, K.-H. (1971): Bericht über die geologischen Gegebenheiten im Bereich des geplanten Wasserwerks Nordheide der Hamburger Wasserwerke GmbH. - 8 S., 13 Anlagen.

Daniels von, C. H. (1977): Abschlußbericht über das Forschungsvorhaben DFG-BE 518/14 „Korrelierung stratigraphischer Einheiten im Nordwestdeutschen Tertiärbecken“. - 50 S., 65 Anl.

Dechend, W., Grimme, K.-H., Groba, E. \& Vierhuff, H. (1972): Karte $1: 500000$ „Tiefenlage der Quartärbasis“ von Nordniedersachsen.

GroвA, E. (1967): Stand der Arbeiten über die Erschließung von Grundwasser im nördlichen Teil der Lüneburger Heide. -4 Anlagen.

-, Ortlam, D. \& Vierhuff, H. (1969): Hydrogeologischer Bericht über die Erschließung von Grundwasser in der Lüneburger Heide. Raum I. - 10 Anlagen. 
Kuster, H. (1973-1976): Wasserwirtschaftliche Rahmenplanung Räume „Untere Elbe“ und „Unterweser-Ost". - Tätigkeitsbericht für 1974/75, 2 Karten, 6 Tabellen. (Tätigkeits-)Berichte über die Räume „Nördlich der Aller“, „Untere Elbe“ und „Unterweser-Ost“ mit Quartärbasiskarten $1: 200000$.

- (1975): Zwischenbericht über die Grundwassererkundung im Raum „Untere Elbe“ und „Unterweser-Ost" der wasserwirtschaftlichen Rahmenplanung - Teilgebiet "Hohe Lieth" zwischen Cuxhaven und Bremerhaven. - 15 S., 9 Anl.

Meyer, K.-J. (1974): Sicherung der Stratigraphie des Jungtertiärs im Nordseebecken mittels palynologischer Untersuchungen. - DFG-Abschlußbericht, 39 S., 7 Anlagen.

OrtLaM, D. (1972): Bericht über Ergebnisse der Grundwasserexplorationsarbeiten im wasserwirtschaftlichen Rahmenplanungsraum „Obere Elbe“ - Zeitabschnitt 1969-1971, 26 S., 134 Anl.

- (1973-1975): Arbeitsberichte über das Forschungsvorhaben „Korrelierung stratigraphischer Einheiten im Nordwestdeutschen Tertiärbecken" — mit Quartärbasiskarten 1:200000. 